\title{
Comparison of SF-36 and WHOQoL-BREF in Measuring Quality of Life in Patients with Type 2 Diabetes
}

This article was published in the following Dove Press journal: International Journal of General Medicine

\begin{abstract}
Abbas Abbasi-Ghahramanloo,' Mojtaba Soltani-Kermanshahi, (iD) ${ }^{2}$ Kamyar Mansori, ${ }^{3}$ Maryam Khazaei-Pool, (iD ${ }^{4,5}$ Masoudreza Sohrabi, ${ }^{6}$ Hamid Reza Baradaran, ${ }^{7,8}$ Zahra Talebloo, ${ }^{9}$ Ali Gholami $^{10,11}$

'Department of Public Health, School of Health, Ardabil University of Medical Sciences, Ardabil, Iran; ${ }^{2}$ Social Determinants of Health Research Center, School of Medicine, Semnan University of Medical Sciences, Semnan, Iran; ${ }^{3}$ Department of Biostatistics and Epidemiology, School of Medicine, Zanjan University of Medical Sciences, Zanjan, Iran; ${ }^{4}$ Department of Public Health, School of Health, Mazandaran University of Medical Sciences, Sari, Iran; ${ }^{5} \mathrm{Health}$ Sciences Research Center, Addiction Research Institute, Mazandaran University of Medical Sciences, Sari, Iran; ${ }^{6}$ Gastrointestinal \& Liver Disease Research Center (GILDRC), Firoozgar Hospital, Iran University of Medical Sciences, Tehran, Iran; ${ }^{7}$ Department of Epidemiology, School of Health, Iran University of Medical Sciences, Tehran, Iran; ${ }^{8}$ Ageing Clinical and Experimental Research Team, Institute of Applied Health Sciences, School of Medicine, Medical Sciences \& Nutrition University of Aberdeen, Aberdeen, UK; ${ }^{9}$ Non-

Communicable Diseases Research Center, Endocrinology and Metabolism Population Sciences Institute, Tehran University of Medical Sciences, Tehran, Iran;

${ }^{10}$ Noncommunicable Diseases Research Center, Neyshabur University of Medical Sciences, Neyshabur, Iran; "'Department of Epidemiology \& Biostatistics, School of Public Health, Neyshabur University of Medical Sciences, Neyshabur, Iran
\end{abstract}

Correspondence: Ali Gholami Janbazan Ave, Neyshabur, Iran Tel +98-5I-42632470 Fax +98-5I-43348895

Email aagholami80@yahoo.com
Objective: This study aimed to compare the quality of life (QoL) in patients with type 2 diabetes using two WHOQoL -BREF and SF-36 questionnaires in Iran.

Patients and Methods: In this cross-sectional study, 1847 patients with type 2 diabetes were recruited from rural health-care centers affiliated to Neyshabur County (Iran) in 2012. In addition to demographic information, two questionnaires WHOQoL-BREF and SF-36 questionnaires were used for data collection. Cronbach's alpha coefficient was used for scale reliability. We conducted an exploratory factor analysis for the investigation of construct validity. Convergent and discriminant validity were analyzed using Spearman correlation coefficient. To determine the relationships between the eight domains of SF-36 and four domains of the WHOQoL-BREF, structural equation modelling was performed.

Results: Cronbach's alpha coefficients were acceptable for all domains of both WHOQoLBREF (0.69-0.86) and SF-36 (0.63 -0.92) questionnaires. The principal component analysis showed two separate factors: one for all domains of SF-36 and another for all domains of WHOQoL-BREF. Spearman correlation coefficients of both instruments were partly to strongly correlated with most domains $(r \geq 0.40)$. Correlations for domains with similar constructs were stronger than those measuring varied constructs. Structural equation modelling recommended approximately moderate relationships among the SF-36 and WHOQoLBREF domains.

Conclusion: Our study suggests that SF-36 and WHOQoL-BREF are reliable instruments for clinical and research uses, respectably. However, results of the goodness of fit showed that the WHOQoL-BREF was fitted well. Also, the WHOQoL-BREF can be considered more suitable for the study population.

Keywords: quality of life, type 2 diabetes, WHOQoL-BREF, SF-36, Iran

\section{Introduction}

Diabetes is a complex metabolic disorder which is determined by hyperglycemia. Hyperglycemia is caused by impaired insulin secretion or function. ${ }^{1}$ Studies show that more than 3 million people have diabetes in Iran. If effective preventive and control measures are not done in this area, it will reach about 7 million people by 2030. Diabetes and its potential complications often lead to high financial costs, reduced quality of life (QoL), and undesirable changes in lifestyle for the patient and his or her family. ${ }^{2}$ Diabetic patients have many physical-psychological problems such as depression, anxiety, disability, inactivity and obesity that ultimately can be lead to a significant decrease in QoL. ${ }^{3,4}$ 
Findings of 20 years of Iranian study revealed an annual crude incidence rate of 10 per 1000 person-year of follow up for type 2 diabetes. ${ }^{5} \mathrm{~A}$ trend analysis showed a $35.1 \%$ increase in diabetes mellitus prevalence from 2005 to 2011 in Iran. ${ }^{6}$ Besides, the economic burden of diabetes mellitus in Iran is predicted to increase markedly in the future decades. ${ }^{7}$

According to the WHO, QoL is defined as an individual's perception of their position in life in the context of the culture and value systems in which they live and in relation to their goals, expectations, standards and concerns. ${ }^{8}$ In recent years, different studies have investigated the QoL of patients with diabetes in Iran and have shown that diabetic patients have a lower QoL than nondiabetic patients, ${ }^{9-11}$ because the diabetes has a profound effect on the QoL of diabetic patients due to physical complications such as retinopathy, nephropathy, neuropathy, cardiovascular disease and mental disorders. ${ }^{12}$ Also, our knowledge shows that most studies in the field have used the Short-Form 36 (SF-36) to assess the QoL of patients with diabetes in Iran. However, some studies have also used the Iranian version of the WHO QoL Questionnaire (WHOQoL-BREF) to investigate the QoL in these patients. But, studies that have simultaneously measured and compared QoL in patients with diabetes using these two questionnaires are very limited, especially in Iran. Regarding the above description, the present study was designed and implemented to compare the QoL in patients with type 2 diabetes using two WHOQoL-BREF and SF-36 questionnaires in Iran. The hypothesis of this study was that both questionnaires (WHOQoL-BREF and SF-36) are reliable for measuring the QoL in patients with diabetes.

\section{Patients and Methods}

\section{Participants}

In our cross-sectional study, 1847 out of the 2224 patients with type 2 diabetes participated in the study and completed the questionnaires. Patients were recruited from rural health-care centers affiliated to Neyshabur University of Medical Sciences (Neyshabur is a city in north-east of Iran) between April and July 2012. Participants were enrolled based on the accessible list in the rural health care centers of Neyshabur. Subjects who (a) had been definitely diagnosed with diabetes type 2, (b) had no coexisting chronic debilitating disease (i.e., stroke, or epilepsy), (c) had no audio-visual or psychological problems, (d) dwelled in Neyshabur rural regions, and (e) accepted to participate in the study. The patients were diagnosed through diabetes screening programs based on fasting blood sugar level. If fasting blood sugar level was $126 \mathrm{mg} / \mathrm{dl}$ or higher on two separate tests, the person detected as a patient with diabetes. To make sure that subjects were not suffering from other chronic diseases or psychological and audio-visual impairments, we asked them some questions and also we reviewed their health records (available in health care centers). Patients $(\mathrm{N}=377)$ with communication difficulties and lack of interest in participating in the study were excluded.

\section{Measures}

To collect data, a three-section scale was applied including a Demographic Data Form (containing of items related to the patients' sociodemographic characteristics-i.e., age, gender, education level, marital status, household income, BMI, and distance from the city), the Iranian validated versions of World Health Organization Quality of Life questionnaire (WHOQoL-BREF), ${ }^{13}$ and the Short-Form 36 (SF-36). ${ }^{14}$

All of three scales were responded by the patients in a single session, first the socio-demographic characteristics, then the WHOQoL-BREF, and finally the SF-36. When requested to help, the investigator was limited to rereading the items slowly. The evaluation was managed by interviewer whenever a patient had problem in understanding the items.

\section{SF-36 Quality of Life Questionnaire}

The SF-36 is one of the most generally used HRQoL scale in the world that has been widely validated in different languages. It is a multicultural scale consisting of 36 questions and categorized into eight-domain profile of scores: physical functioning (PF; 10 items), general health (GH; 5 items), role physical (i.e., role limitations due to the physical health problems, RP; 4 items), bodily pain (BP; 2 items), social functioning (SF; 2 items), vitality (VT; 4 items), role emotional (i.e., role limitations due to emotional problems, RE; 3 items), and mental health (MH; 5 items). ${ }^{15}$ For each domain, a score ranging from 0 to 100 was assessed with a higher score indicating better health. For the Iranian version of the SF-36, acceptable internal consistency (Cronbach's alpha coefficients were greater from 0.77 to 0.90 ) and construct validities (discriminant and convergent validities) have been reported above 0.40 ranging from 0.58 to $0.95 .{ }^{14}$ 


\section{WHOQoL-BREF Quality of Life Scale}

The WHOQoL-100 is a multidimensional scale developed for applying with a wide range of psychological and physical disorders. The WHOQoL Group initially generated a WHOQoL scale with a 100-question form that allowed a comprehensive estimation of 24 aspects about quality of life. Having said that, the long content of questionnaire made it difficult for researchers to assess only the HRQoL. Consequently, two questions from the total health and general QoL and one question from each of the remaining twenty-four aspects were chosen to form the WHOQoLBREF. ${ }^{16}$ These aspects are classified into four domains: Physical health (PH; 7 items), psychological well-being (PS; 6 items), social relationships (SR; 3 items) and environment health (EH; 8 items). All questions are rated on a 5-point Likert scale, and the item is scored between 1 and 5. Raw scores in each domain were changed to a 4-20 score based on the guideline. All domain scores were linearly changed such that they varied from 0 to 100 with " 100 " demonstrating the highest possible QoL. The Iranian version of the WHOQoL-BREF was validated in compliance with WHO guidelines. ${ }^{17}$ Additionally, comprising 26-question translated from the original WHOQoL-BREF questionnaire, the Iranian version contains two added questions of local importance, i.e., Being Valued and Food Availability. ${ }^{18}$ Good reliabilities (including an internal consistency of 0.$76-0.82$ and a test-retest reliability of $0.76-0.82$ ) and validities of the Iranian version have been founded. ${ }^{19}$ To use all information in the WHOQoL-BREF, the Overall Health and General QoL aspect also were assessed as a single score as with the scoring method for the other domain scores, although this single aspect score was not applied by the WHOQoL group.

\section{Statistical Analysis Exploratory Factor Analysis}

Exploratory factor analysis was performed to test construct validity, using a principal component analysis with varimax rotation approach for the 12 separate domains (4 domains from the WHOQoL-BREF and 8 domains from the SF-36). Eigenvalues greater than $1(>1)$ and scree plot were performed to determine the number of quality of life factors extracted. Factor loadings equal to or more than 0.4 were determined suitable. ${ }^{20}$

\section{Reliability}

Internal consistency of the domains was assessed by the Cronbach's alpha coefficient for the entire scales, each construct, and each factor. The Cronbach's alpha coefficient of 0.7 or above was determined acceptable. ${ }^{21}$

\section{Convergent \& Discriminant Validity}

To evaluate the convergence and discriminant validity, correlations among the SF-36 and the WHOQoL-BREF were examined applying Spearman correlation coefficient. It was hypothesized that those domains that are conceptually related would be more strongly correlated, but those domains in the two instruments with less in common would demonstrate weaker correlations.

Therefore, we assumed moderate to high correlations $(r \geq 0.4)$ between all domains of the WHOQoL-BREF and all domains of the SF-36; the Overall QoL and GH of the WHOQoL-BREF and the GH of the SF-36; the PF, RP, and BP domains of the SF-36 with consisting domain the $\mathrm{PH}$ of the WHOQoL-BREF; the SF, RE, and MH domains of the SF-36 with consisting domains and the PS and SR of the WHOQoL-BREF.

\section{Confirmatory Factor Analysis}

To examine the relationships between the eight domains of SF-36 and four domains of the WHOQoL-BREF, structural equation modeling was performed. It was tested the extent to which the variance in each domain was explained by other domains in both instruments. We used the incremental fit index (IFI), the comparative fit index (CFI), the Tucker-Lewis Index (TLI), the root-mean-square error of approximation (RMSEA), and the Akaike's information criterion (AIC) in order to assess model fit. AMOS software version 23 was used for structural equation modeling and $p<0.05$ was considered as statistically significant.

\section{Ethical Considerations}

This study was approved by the Ethical Committee of Iran university of medical sciences approved the study (Ethical code: IR.IUMS.REC 1394.94-02-193-26156). Permission to conduct the study was obtained from this committee and the study was conducted in accordance with the Declaration of Helsinki. A verbal informed consent form was received from all the participants before initiation of the study.

\section{Results Patient Characteristics}

1847 out of all 2224 patients with type 2 diabetes were included in the study. There were 1289 female (69.8\%) and 558 male $(30.2 \%)$ patients in the study group. The 
mean $\pm \mathrm{SD}$ age was $59.65 \pm 12.3$ years, and the age range was $30-97$ years. Of the patients, $19.9 \%$ were married, $66.2 \%$ were Illiterate, and household salary of $86.9 \%$ of patients was less than $200 \$$ per months (Table 1).

Score distributions of both the SF-36 and the WHOQoL-BREF are presented in Table 2. Of all the subscales, SF achieved the highest value for SF-36 and

Table I Characteristics of Study Population $(n=1847)$

\begin{tabular}{|l|l|l|}
\hline Characteristics & $\mathbf{n}$ & $\%$ \\
\hline Sex & 558 & \\
Male & 1289 & 30.2 \\
Female & & 69.8 \\
\hline Age* & 922 & \\
$<60 \mathrm{yr}$ & 921 & 50 \\
$\geq 60 \mathrm{yr}$ & & 50 \\
\hline Education Level* & 1203 & \\
Illiterate & 613 & 66.2 \\
$\geq$ Elementary & & 33.8 \\
\hline Marital Status & 1480 & \\
Single/Divorced & 367 & 80.1 \\
Married & & 19.9 \\
\hline Household Income (per month)* & 1272 & 86.9 \\
$<200$ \$ & 192 & 13.1 \\
\hline 2200 \$ &
\end{tabular}

Note: *Some data were missing (4 data in age and 31 data in education level).
SR for WHOQoL-BREF. The lowest was GH for SF-36 and PS for WHOQoL-BREF.

\section{Scale Reliability}

The internal consistency of all domains is presented on the diagonal of Table 2. Cronbach's alpha coefficients for SF36 subscales ranged from $0.63(\mathrm{SF})$ to $0.92(\mathrm{PF})$, and from 0.69 (SR) to $0.86(\mathrm{PH})$ for the WHOQoL-BREF showing good internal consistency between these specific items within each domain.

\section{Construct Validity}

It was applied iterated principal component analysis with oblique rotation to specify the structure of all of the domains for the SF-36 and WHOQoL-BREF. It was found two separate factors with eigenvalues more than 1.0 (Table 3). However, the scree plot revealed a threefactor solution. Furthermore, the first and second factors accounted for $50.9 \%$ and $10.4 \%$ of the variance in all domains. The proper level for a factor was considered acceptable when they loaded equal or greater than 0.40 . The first factor extracted included all domains of the SF36 , and the second factor extracted included all domains of the WHOQoL-BREF.

\section{Convergent and Discriminant Validity}

In Spearman correlation coefficients of the SF-36, domains assessing similar constructs were partly to moderately correlated $(\mathrm{r}=0.40-0.60)$, while domains assessing varied

Table 2 Score Distributions of the Instruments SF-36 and WHOQoL-100

\begin{tabular}{|c|c|c|c|c|c|c|c|c|}
\hline Subscales & Mean & SD & Median & Minimum Score & $\begin{array}{l}\text { Maximum } \\
\text { Score }\end{array}$ & Lower Quartile & Upper Quartile & Cronbach's $\alpha$ \\
\hline \multicolumn{9}{|l|}{ SF-36 } \\
\hline PF & 54.99 & 28.50 & 55 & 0 & 100 & 35 & 75 & 0.92 \\
\hline $\mathrm{RP}$ & 46.63 & 42.92 & 50 & 0 & 100 & 0 & 100 & 0.88 \\
\hline $\mathrm{BP}$ & 54.43 & 23.23 & 55 & 0 & 100 & 45 & 67.50 & 0.83 \\
\hline $\mathrm{GH}$ & 39.89 & 18.13 & 40 & 0 & 100 & 30 & 50 & 0.76 \\
\hline VT & 54.94 & $|7.7|$ & 56 & 0 & 100 & 35 & 60 & 0.73 \\
\hline SF & 62.18 & 21.02 & 62.5 & 0 & 100 & 50 & 75 & 0.63 \\
\hline RE & 50.37 & 44.90 & 33.33 & 0 & 100 & 0 & 100 & 0.88 \\
\hline $\mathrm{MH}$ & 47.87 & 18.76 & 50 & 0 & 100 & 44 & 68 & 0.77 \\
\hline \multicolumn{9}{|l|}{ WHOQOL-BREF } \\
\hline $\mathrm{PH}$ & 49.33 & 16.79 & 50 & 0 & 100 & 38 & 63 & 0.86 \\
\hline PS & 48.39 & 15.58 & 50 & 0 & 100 & 44 & 56 & 0.78 \\
\hline$S R$ & 54.16 & 18.39 & 56 & 0 & 100 & 44 & 69 & 0.69 \\
\hline $\mathrm{EH}$ & 52.89 & 13.73 & 56 & 0 & 100 & 44 & 63 & 0.76 \\
\hline
\end{tabular}

Abbreviations: SF-36, Short Form 36 Health Survey; WHOQoL-BREF, World Health Organization Quality of Life Scale Brief Version; SD, standard deviation; PF, physical functioning; RP, role physical; BP, bodily pain; GH, general health; VT, vitality; SF, social functioning; RE, role emotional; MH, mental health. PH, physical health; PS, psychological well-being; SR, social relationships; EH, environment health. 
Table 3 Exploratory Factor Analysis ${ }^{\mathrm{a}}$ of the SF-36 and WHOQoL-BREF

\begin{tabular}{|c|l|l|l|}
\hline \multirow{2}{*}{ Subscales } & \multicolumn{2}{|l|}{ Factor Loading } & \multirow{2}{*}{$\mathbf{H}^{\mathbf{2 c}}$} \\
\cline { 2 - 3 } & Factor I & Factor II & \\
\hline SF-36 & & & \\
PF & 0.683 & 0.210 & 0.510 \\
RP & 0.803 & 0.057 & 0.649 \\
BP & 0.737 & 0.264 & 0.613 \\
GH & 0.635 & 0.435 & 0.593 \\
VT & 0.593 & 0.563 & 0.669 \\
SF & 0.523 & 0.383 & 0.420 \\
RE & 0.713 & 0.168 & 0.536 \\
MH & 0.593 & 0.497 & 0.599 \\
\hline WHOQoL-BREF & & & \\
PH & 0.583 & 0.637 & 0.746 \\
PS & 0.320 & 0.808 & 0.756 \\
SR & 0.139 & 0.736 & 0.561 \\
EH & 0.092 & 0.829 & 0.695 \\
\hline
\end{tabular}

Notes: ${ }^{\text {aU }}$ sing iterated principal factor analysis with eigenvalue $>1.0$. ${ }^{\mathrm{b}}$ After oblique rotation. 'Communality: proportion of a variable's variance explained by a factor structure.

Abbreviations: SF-36, Short Form 36 Health Survey; WHOQoL-BREF, World Health Organization Quality of Life Scale Brief Version; PF, physical functioning; $\mathrm{RP}$, role physical; BP, bodily pain; GH, general health; VT, vitality; SF, social functioning; RE, role emotional; $\mathrm{MH}$, mental health. PH, physical health; PS, psychological well-being; SR, social relationships; EH, environment health.

constructs were weakly correlated $(\mathrm{r}<0.40)$. For example, the correlation between PF and RP was 0.49, but the correlation of $\mathrm{PF}$ and $\mathrm{MH}$ was 0.37 . Correlations between the WHOQoL-BREF domains were reasonable. For domains that assess like concepts in both the SF-36 and WHOQoL-BREF, the PF, SF, and MH domains of the SF36 were moderately correlated $(\mathrm{r}=0.40-0.60)$ with the corresponding PH and SR domains of the WHOQoL-BREF (Table 4). For instance, the correlation between the SF-36 $\mathrm{PF}$ domain and the WHOQoL-BREF PH domain was 0.55 ; the association of the SF-36 MH domain and the WHOQoL-BREF PS domain was 0.6. Domains in the SF36 and WHOQoL-BREF that did not match to one another were similarly associated (Table 4). For instance, the association of the SF-36 PF domain and the WHOQoL-BREF PS domain was 0.37 .

\section{Relations Among Domains of Both SF-36 and WHOQoL-BREF}

The eight domains of SF-36 and four domains of WHOQoL-BREF were also subjected to the confirmatory factor analysis to test the fitness of a model. This model fits the relationships among all domains of the SF-36 and
WHOQoL-BREF good. Covariance matrixes were applied and fit indexes were calculated. The relative chi-square $(\chi 2)$ for models 1,2 , and 3 was equal to $24.29,7.23$, 263.16, respectively, and showing the goodness of fit for the model $(\mathrm{P}<0.05)$. The RMSEA of model 1 was 0.028 presenting good fit. Additionally, the RMSEA for models 2 and 3 was 0.058 and 0.06 , respectively. All comparative indices of the 3-model including CFI, IFI, and TLI were more than 0.90 (0.998, 0.998, and 0.994 for model 1 , $0.998,0.998$, and 0.988 for model 2 , and $0.981,0.981$, and 0.964 for model 3, respectively) of which fall in the acceptable range. The AIC for models 1, 2, and 3 were $72.0,20.0$ and 156.0, respectively, showing adequate fit for three models (Table 5). The results obtained from the CFA are shown in Figures 1 and 2 and Table 5. Comparing Figures 1 and 2 showed that weights of subscales were not statistically different with weights in separate models (P-value $=0.507)$.

\section{Discussion}

In this population from Iran (Neyshabur) patients with type 2 diabetes, the SF-36 and WHOQoL-BREF questionnaires appeared to measure distinct concepts related to QoL. In our study sample, about $90 \%$ of patients' household income was lower than $200 \$$ per month and that was approximately approved with sex ratio and education level ratio. It is discovered that social subscales are high with two instruments. It can be argued that the questions of the two subscales are very similar; SF has 2 items and SR have 3 items. The exploratory factor analysis approximately extracted two distinct factors influencing QoL one factor was associated with all subscales of the SF-36 and the other one associated with all subscales of the WHOQoL-BREF. Our results are consistent with a study carried out by Huang et al. ${ }^{22}$ In the multitrait analysis, correlations were approximately similar among subscales hypothesized to measure homogenous constructs and heterogeneous subscales. Our results suggested that the questionnaires related domains approximately provided similar measures in evaluating Iranian (Neyshabur) patients with type 2 diabetes and Structural equation modelling suggested approximately moderate relationships among domains of the SF-36 and WHOQoL-BREF. The correlation between the SF-36 and the WHOQoL-BREF has been examined in some studies and in different fields. In a national survey on 11,440 people, Huang et al indicated that the correlations were weak among the subscales of both instruments and concluded that both SF-36 and 
Table 4 Correlation Coefficients Between the SF-36 and WHOQoL-BREF Domains

\begin{tabular}{|c|c|c|c|c|c|c|c|c|c|c|c|c|c|}
\hline & & PF & $\mathbf{R P}$ & RE & VT & MH & SF & BP & GH & PH & PS & SR & EH \\
\hline \multirow[t]{2}{*}{ PF } & $\mathrm{CC}$ & 1 & 0.49 & 0.40 & 0.46 & 0.37 & 0.36 & 0.50 & 0.48 & 0.55 & 0.37 & 0.31 & 0.3 \\
\hline & Sig & & $<0.001$ & 0.005 & $<0.001$ & $<0.001$ & $<0.001$ & $<0.001$ & $<0.001$ & $<0.001$ & $<0.001$ & $<0.001$ & $<0.001$ \\
\hline \multirow[t]{2}{*}{$\mathrm{RP}$} & $\mathrm{CC}$ & & 1 & 0.65 & 0.41 & 0.36 & 0.33 & 0.49 & 0.43 & 0.48 & 0.35 & 0.27 & 0.25 \\
\hline & Sig & & & $<0.001$ & $<0.001$ & $<0.001$ & $<0.001$ & $<0.001$ & $<0.001$ & $<0.001$ & $<0.001$ & $<0.001$ & $<0.001$ \\
\hline \multirow[t]{2}{*}{ RE } & $\mathrm{CC}$ & & & I & 0.45 & 0.43 & 0.37 & 0.41 & 0.39 & 0.45 & 0.40 & 0.31 & 0.30 \\
\hline & Sig & & & & $<0.001$ & $<0.001$ & $<0.001$ & $<0.001$ & $<0.001$ & $<0.001$ & $<0.001$ & $<0.001$ & $<0.001$ \\
\hline \multirow[t]{2}{*}{ VT } & $\mathrm{CC}$ & & & & 1 & 0.74 & 0.48 & 0.55 & 0.68 & 0.66 & 0.59 & $0.4 I$ & 0.43 \\
\hline & Sig & & & & & $<0.001$ & $<0.001$ & $<0.001$ & $<0.001$ & $<0.001$ & $<0.001$ & $<0.001$ & $<0.001$ \\
\hline \multirow[t]{2}{*}{$\mathrm{MH}$} & $\mathrm{CC}$ & & & & & & 0.52 & 0.48 & 0.56 & 0.57 & 0.60 & 0.41 & 0.42 \\
\hline & Sig & & & & & & $<0.001$ & $<0.001$ & $<0.001$ & $<0.001$ & $<0.001$ & $<0.001$ & $<0.001$ \\
\hline \multirow[t]{2}{*}{ SF } & $\mathrm{CC}$ & & & & & & I & 0.50 & 0.40 & 0.49 & 0.44 & 0.32 & 0.30 \\
\hline & Sig & & & & & & & $<0.001$ & $<0.001$ & $<0.001$ & $<0.001$ & $<0.001$ & $<0.001$ \\
\hline \multirow[t]{2}{*}{ BP } & $\mathrm{CC}$ & & & & & & & 1 & 0.58 & 0.62 & 0.43 & 0.29 & 0.28 \\
\hline & Sig & & & & & & & & $<0.001$ & $<0.001$ & $<0.001$ & $<0.001$ & $<0.001$ \\
\hline \multirow[t]{2}{*}{$\mathrm{GH}$} & $\mathrm{CC}$ & & & & & & & & 1 & 0.67 & $0.5 \mathrm{I}$ & 0.34 & 0.36 \\
\hline & Sig & & & & & & & & & $<0.001$ & $<0.001$ & $<0.001$ & $<0.001$ \\
\hline \multirow[t]{2}{*}{$\mathrm{PH}$} & $\mathrm{CC}$ & & & & & & & & & 1 & 0.70 & 0.49 & 0.56 \\
\hline & Sig & & & & & & & & & & $<0.001$ & $<0.001$ & $<0.001$ \\
\hline \multirow[t]{2}{*}{ PS } & $\mathrm{CC}$ & & & & & & & & & & & 0.53 & 0.67 \\
\hline & Sig & & & & & & & & & & & $<0.001$ & $<0.001$ \\
\hline \multirow[t]{2}{*}{ SR } & $\mathrm{CC}$ & & & & & & & & & & & I & 0.53 \\
\hline & Sig & & & & & & & & & & & & $<0.001$ \\
\hline $\mathrm{EH}$ & $\mathrm{CC}$ & & & & & & & & & & & & \\
\hline & Sig & & & & & & & & & & & & \\
\hline
\end{tabular}

Abbreviations: SF-36, Short Form 36 Health Survey; WHOQoL-BREF, World Health Organization Quality of Life Scale Brief Version; PF, physical functioning; RP, role physical; BP, bodily pain; GH, general health; VT, vitality; SF, social functioning; RE, role emotional; MH, mental health. PH, physical health; PS, psychological well-being; SR, social relationships; EH, environment health; CC, correlation coefficients; Sig, significant. (2-tailed).

Table 5 Three Used Models and the Fitness of a Models

\begin{tabular}{|l|l|l|l|}
\hline \multirow{2}{*}{ Measure } & \multicolumn{3}{|l|}{ Model } \\
\cline { 2 - 4 } & $\mathbf{I}$ & $\mathbf{2}$ & $\mathbf{3}$ \\
\hline$\chi^{2}$ & 24.29 & 7.23 & 263.16 \\
df & 10 & 1 & 34 \\
P-value & 0.007 & 0.007 & $<0.00 \mathrm{I}$ \\
CFI & $\mathbf{0 . 9 9 8}$ & $\mathbf{0 . 9 9 8}$ & $\mathbf{0 . 9 8 I}$ \\
IFI & $\mathbf{0 . 9 9 8}$ & $\mathbf{0 . 9 9 8}$ & $\mathbf{0 . 9 8 I}$ \\
TLI & $\mathbf{0 . 9 9 4}$ & $\mathbf{0 . 9 8 8}$ & $\mathbf{0 . 9 6 4}$ \\
RMSEA & $\mathbf{0 . 0 2 8}$ & $\mathbf{0 . 0 5 8}$ & $\mathbf{0 . 0 6}$ \\
AIC & 72.0 & 20.0 & 156.0 \\
\hline
\end{tabular}

Notes: Model I construct of SF-36; Model 2 construct of WHOQoL- BREF; Model 3 correlated methods (i.e., SF-36 and WHOQoL-BREF). Acceptable fit indices are in bold.

Abbreviations: $\chi^{2}$, chi-square; $\mathrm{df}$, degree of freedom; IFI, incremental fit index; CFI, comparative fit index; TLI, Tucker-Lewis Index; RMSEA, root-mean-square error of approximation; SRMR, standardized root-mean-square residual; AIC, Akaike's information criterion; SF-36, Short Form 36 Health Survey; WHOQoLBREF, World Health Organization Quality of Life Scale Brief Version.

WHOQoL-BREF appeared to measure different constructs. To put it another way, the SF-36 measured healthrelated QoL, while the WHOQoL-BREF measured global QoL. ${ }^{22}$ However, in another study by Hsiung et al on patients with HIV infection, both the WHOQoL-BREF and the SF-36 were reliable and valid health-related QoL instruments. ${ }^{23}$ In the present study, we found moderate correlations across the subscales of the two questionnaires among type 2 diabetes patients. It appears that the reliability and validity of these two questionnaires for the evaluation of QoL in patients with variant diseases may be different. Transversal studies have shown that the SF36 is a valid and reliable instrument for detecting differences between groups defined by age, sex, socio-economic status, and clinical condition. ${ }^{24}$ Also, in a study among the Iranian population, the Persian version of the SF-36 performed well and the findings suggested that it was a reliable and valid measure of QoL among the general populations. ${ }^{14}$ Therefore, it can be concluded that SF-36 is an applicable tool for the evaluation of QoL in patients with type 2 diabetes. The correlations between the WHOQoL-BREF and the SF-36 were as expected in most items. A correlation higher than 0.45 was found between the physical domain of the WHOQoL-BREF and emotional role, vitality, mental health and social 

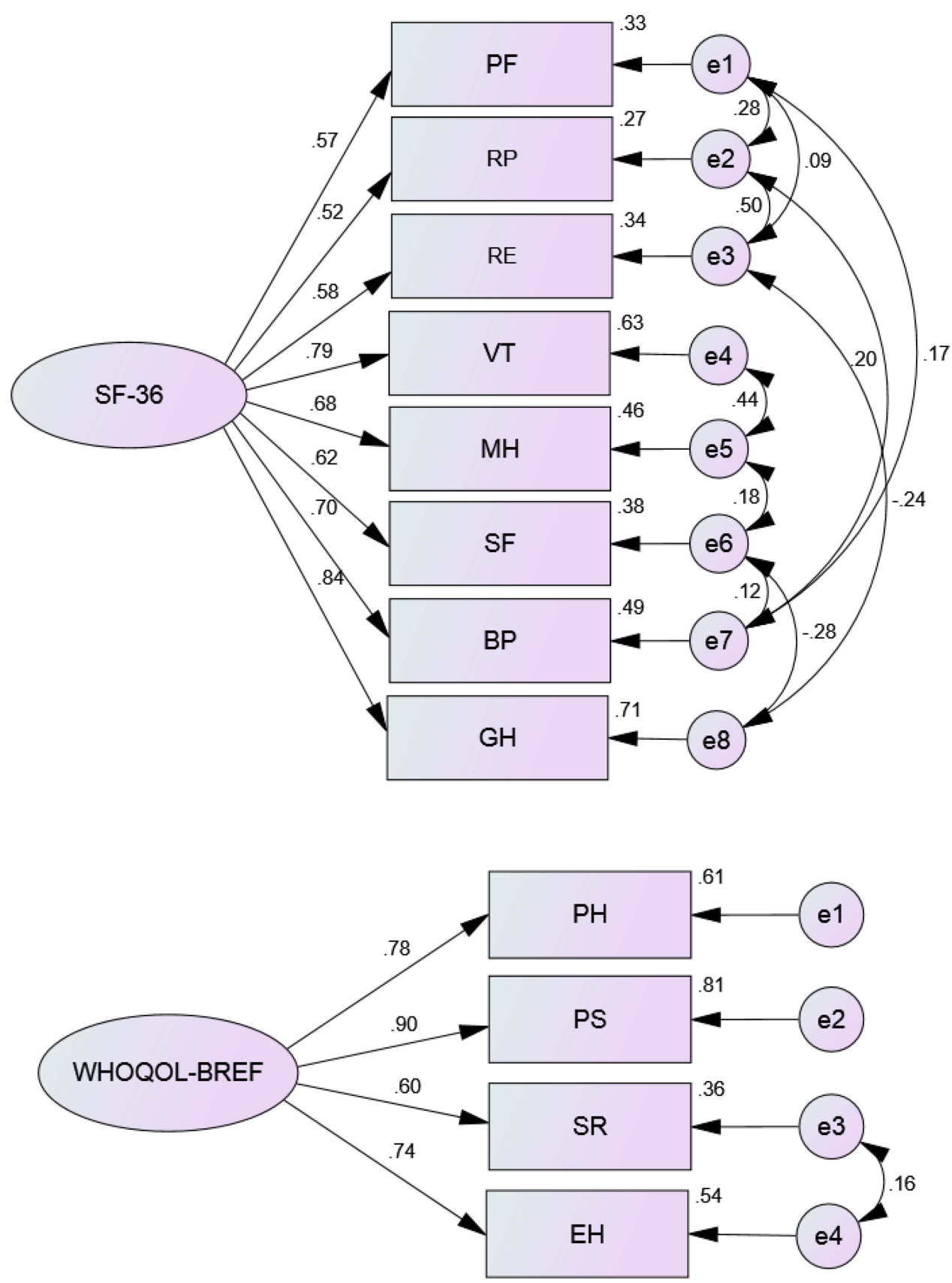

Figure I (A) Model I: Confirmatory factor analysis (CFA) for the eight domains of the SF-36. (B) Model 2: Confirmatory factor analysis (CFA) for the four domains of the WHOQoL-BREF $(\mathrm{n}=1847)$.

Abbreviations: SF-36, Short Form 36 Health Survey; WHOQoL-BREF, World Health Organization Quality of Life Scale Brief Version; PF, Physical Functioning; GH, General Health; RP, Role Physical; BP, Bodily Pain; SF, Social Functioning; VT, Vitality; RE, Role Emotional; MH, Mental Health. PH, Physical Health; PS, Psychological well-being; SR, Social Relationships; EH, Environment Health.

functioning subscales from the SF-36 that were more than the expected size. This can be attributed to overlapping domains in the WHOQoL-BREF. ${ }^{25,26}$ Bonomi et $\mathrm{al}^{26}$ reported that physical health subscales (PF, RP, BP, and $\mathrm{GH}$ ) of the SF-36 were moderately correlated (r: 0.6-0.4) with both physical and psychological subscales of the WHOQoL-BREF. In our study, some mental subscales
(VT and MH) of the SF-36 were highly correlated $(r>0.6)$ with both physical and psychological subscales of the WHOQoL-BREF, and some mental subscales (SF and RE) were moderately correlated with both physical and psychological WHOQoL-BREF subscales. Norholm ${ }^{27}$ and Skevington ${ }^{28}$ reported that physical subscales of the SF-36 were more strongly correlated with physical than 


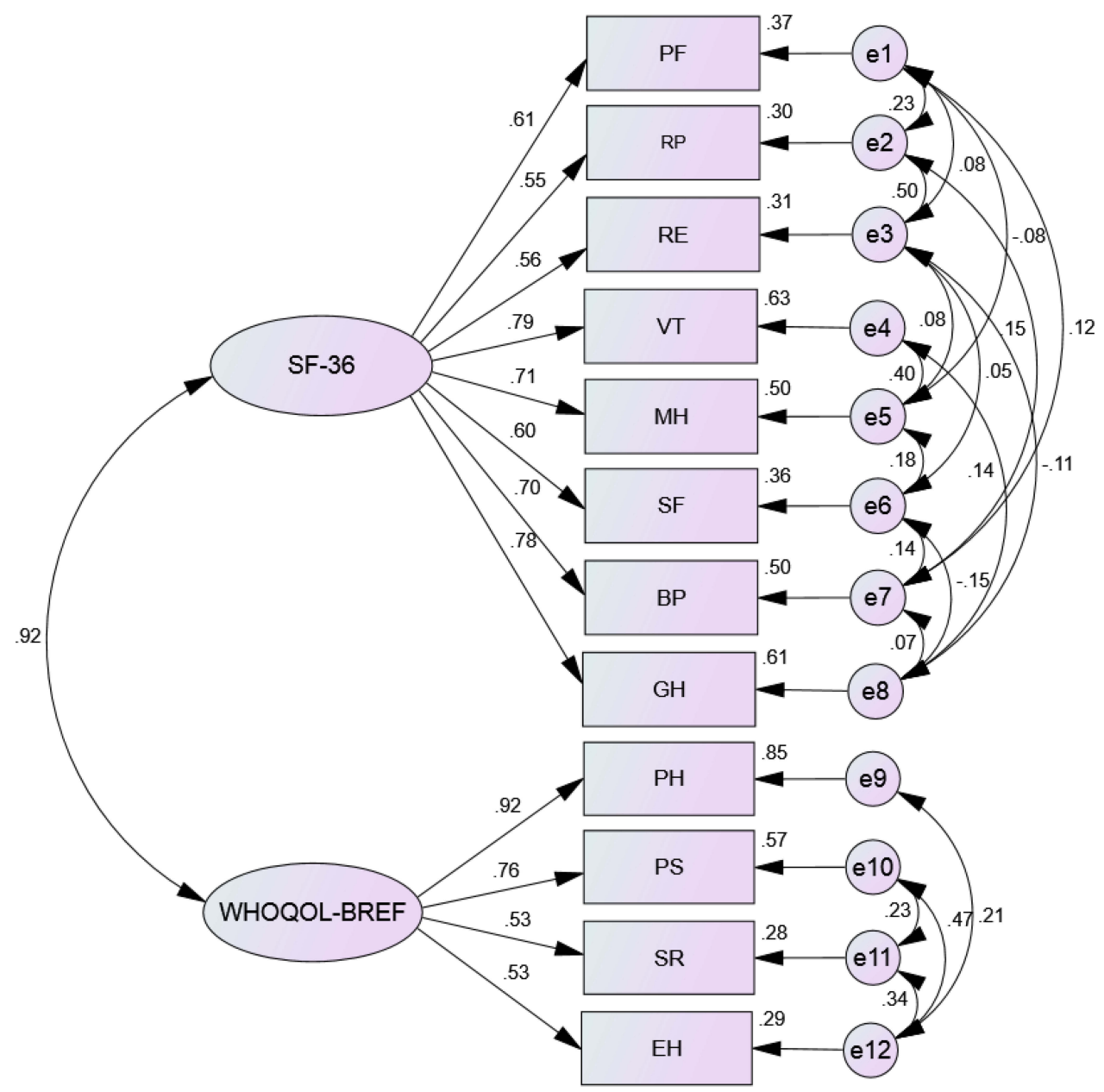

Figure 2 Model 3: Confirmatory factor analysis (CFA) for the eight domains of the SF-36 in correlation to four domains of the WHOQoL-BREF ( $\mathrm{W}=$ I847).

Abbreviations: SF-36, Short Form 36 Health Survey; WHOQoL-BREF, World Health Organization Quality of Life Scale Brief Version; PF, Physical Functioning; GH, General Health; RP, Role Physical; BP, Bodily Pain; SF, Social Functioning; VT, Vitality; RE, Role Emotional; MH, Mental Health. PH, Physical Health; PS, Psychological well-being; SR, Social Relationships; EH, Environment Health.

psychological subscales of the WHOQoL-BREF. Furthermore, it is likely that SF-36 is a more objective measure, because its questions are about capability and disability, whereas WHOQoL-BREF focuses on individual opinions about QoL and so the SF-36 questions are more objective in comparison to the completely subjective questions of the WHOQoL-BREF. However, evidence suggests that these tools do not measure exactly the same constructs. The evidence suggests that WHOQoL-BREF is more sensitive to the demographic characteristics of participants. ${ }^{22,29}$ The literature indicates that these instruments have a weak convergent validity for healthy populations ${ }^{22,30}$ and high magnitude correlation for studies with patient groups. ${ }^{23,31}$ The observed divergences may be a consequence of differences in the goals adopted by developers of the instruments. SF-36 measures aspects that are linked to health and functional performance of individuals, whereas WHOQoL-BREF instruments attempt to measure a broad range of factors concerning the organism, task, and environment. ${ }^{32,33}$

In construct validity, we also used correlations of SF36 and WHOQoL-BREF and results showed that weights of subscales were not statistically different with weights in separate models. However, in some subscales (especially in WHOQoL-BREF subscales) there were seen more than $15 \%$ changes.

We also compared the goodness of fit of three models with different indexes. We found that approximately all three models were fitted well and similarly. But according to AIC index, model 2 was fitted well. Both scales represented acceptable reliability in this study. The study findings indicated satisfactory alpha coefficients in all domains except for the SF and SR. This can be attributed to the small number of questions (2 items in SF-36 and 3 items 
in WHOQoL-BREF) in their domains. In addition, these domains do not appear very homogenous at least in the Iranian culture, since they inquire about sexual life and social supports that are relatively different concepts in Iranian culture. Other studies conducted upon different populations showed similar results concerning reliability. $^{22,23,30,31,34-36}$ It is important to make sure that the results can be generalized to evaluate a particular patient, because the statistical analyses of these studies are based on large samples and do not consider individual differences.

There are some explanations for differences between these two questionnaires. First, SF-36 discriminates better among different levels of health status and utilization than the WHOQoL-BREF. As a result, it may be appropriate to describe the SF-36 as a measure of health-related QoL that focus mainly on health-related functioning and perceptions. Second, same subscales of the two instruments may measure different concepts of perceived QoL. In other words, the SF-36 may measure objective QoL. However, the WHOQoL-BREF may measure self-reported subjective QoL. ${ }^{22}$

However, some limitations should be mentioned. First, the generalizability of our findings may be limited because all patients in this study were recruited from rural regions. The study was unable to enroll representative patients with diabetes in urban regions. Second, cross-sectional design was used in this study. Prospective studies are needed to specify the responsiveness of studied instruments based on changes in the clinical status of patients with diabetes.

In conclusion, this study suggests that SF-36 and WHOQoL-BREF are reliable for clinical and research uses. But according to the goodness of fit results, the WHOQoL-BREF showed a better fit. Moreover, the WHOQoL-BREF can be considered more suitable for this study population because it valued age in individuals' personal opinions. Nevertheless, to select one, researchers should consider which aspects of QoL they aim to capture, because of the observed weak convergent validity signs.

\section{Disclosure}

The authors report no conflicts of interest in this work.

\section{References}

1. Barnard KD, Lloyd CE, Holt RI. Psychological burden of diabetes and what it means to people with diabetes. Psychol Diabetes Care. 2012;1-22.
2. West SP, Lagua C, Trief PM, Izquierdo R, Weinstock RS. Goal setting using telemedicine in rural underserved older adults with diabetes: experiences from the informatics for diabetes education and telemedicine project. Telemedicine e-Health. 2010;16 (4):405-416. doi:10.1089/tmj.2009.0136

3. Ghavami H, Ahmadi F, Entezami H, Meamarian R. The effect of continuous care model on diabetic patients' blood pressure. Iran $J$ Med Educ. 2006;6(2):87-95.

4. Zareipour M, Ghelichi Ghojogh M, Mahdi-akhgar M, Alinejad M, Akbari S. The quality of life in relationship with glycemic control in people with Type2 diabetes. J Commun Health Res. 2017;6(3):141-149.

5. Ramezankhani A, Harati H, Bozorgmanesh M, et al. Diabetes mellitus: findings from 20 years of the Tehran lipid and glucose study. Int J Endocrinol Metabol. 2018;16(4Suppl). doi:10.5812/ijem.84784.

6. Esteghamati A, Etemad K, Koohpayehzadeh J, et al. Trends in the prevalence of diabetes and impaired fasting glucose in association with obesity in Iran: 2005-2011. Diabetes Res Clin Pract. 2014;103 (2):319-327. doi:10.1016/j.diabres.2013.12.034

7. Javanbakht M, Mashayekhi A, Baradaran HR, Haghdoost A, Afshin A. Projection of diabetes population size and associated economic burden through 2030 in Iran: evidence from micro-simulation Markov model and Bayesian meta-analysis. PLoS One. 2015;10:7. doi:10.1371/journal.pone.0132505

8. Dimitrova B, Carr AJ, Higginson IJ, Robinson PG. Quality of life. London: BMJ Books, 2003. 133 pp. ISBN 0-7279-1544-4. Eur J Public Health. 2005;15(6):668-+. doi:10.1093/eurpub/cki178

9. Baghiani Moghadam MAAM, Mazlumi S, Saeedi Zade MA. Survey on the quality of life in type 2diabetes patients Yazd. J Yazd Univ Med Sci. 2006;4(4):49-54.

10. Kiadaliri AA, Najafi B, Mirmalek-Sani M. Quality of life in people with diabetes: a systematic review of studies in Iran. J Diabetes Metab Disord. 2013;12(1):54. doi:10.1186/2251-6581-12-54

11. Javanbakht M, Abolhasani F, Mashayekhi A, Baradaran HR. Health related quality of life in patients with type 2 diabetes mellitus in Iran: a national survey. PLoS One. 2012;7(8):8. doi:10.1371/journal. pone. 0044526

12. Longo DL, Fauci AS, Kasper DL, Hauser SL, Jameson JL, Loscalzo J. Harrison's Principles of Internal Medicine. New York: Mcgraw-hill; 2012.

13. Nejat S, Montazeri A, Holakoui.e.Naieni K, Mohammad K, Majdzadeh S. The World Health Organization quality of Life (WHOQOL-BREF) questionnaire: translation and validation study of the Iranian version. J School Publ Health Inst Public Health Res. 2006;4(4):1-12.

14. Montazeri A, Goshtasebi A, Vahdaninia M, Gandek B. The Short Form Health Survey (SF-36): translation and validation study of the Iranian version. Qual Life Res. 2005;14(3):875-882. doi:10.1007/ s11136-004-1014-5

15. Ware JE Jr., Sherbourne CD, The MOS. 36-item short-form health survey (SF-36). I conceptual framework and item selection . Med Care. 1992;30(6):473-483.

16. The WHOQOL Group. Development of the World Health Organization WHOQOL-BREF quality of life assessment. Psychol Med. 1998;28(3):551-558. doi:10.1017/S0033291798006667

17. Study protocol for the World Health Organization project to develop a Quality of Life assessment instrument (WHOQOL). Qual Life Res. 1993;2(2):153-159. doi:10.1007/BF00435734

18. !!! INVALID CITATION !!! (17).

19. Yousefy AR, Ghassemi GR, Sarrafzadegan N, Mallik S, Baghaei AM, Rabiei K. Psychometric properties of the WHOQOL-BREF in an Iranian adult sample. Community Ment Health J. 2010;46(2):139-147. doi:10.1007/s10597-009-9282-8

20. Gaskin CJ, Happell B. On exploratory factor analysis: a review of recent evidence, an assessment of current practice, and recommendations for future use. Int J Nurs Stud. 2014;51(3):511-521. doi:10. 1016/j.ijnurstu.2013.10.005 
21. Novick MR, Lewis C. Coefficient alpha and the reliability of composite measurements. Psychometrika. 1967;32(1):1-13. doi:10.1007/ BF02289400

22. Huang I-C, Wu AW, Frangakis C. Do the SF-36 and WHOQOL-BREF measure the same constructs? Evidence from the Taiwan population. Qual Life Res. 2006;15(1):15-24. doi:10.1007/ s11136-005-8486-9

23. Hsiung P-C, Fang C-T, Chang -Y-Y, Chen M-Y, Wang J-D. Comparison of WHOQOL-BREF and SF-36 in patients with HIV infection. Qual Life Res. 2005;14(1):141-150. doi:10.1007/s11136004-6252-z

24. Failde I, Ramos I. Validity and reliability of the SF-36 Health Survey Questionnaire in patients with coronary artery disease. $J$ Clin Epidemiol. 2000;53(4):359-365. doi:10.1016/S0895-4356 (99)00175-4

25. Nedjat S, Montazeri A, Holakouie K, Mohammad K, Majdzadeh R. Psychometric properties of the Iranian interview-administered version of the World Health Organization's Quality of Life Questionnaire (WHOQOL-BREF): a population-based study. BMC Health Serv Res. 2008;8(1):61. doi:10.1186/1472-6963-8-61

26. Bonomi AE, Patrick DL, Bushnell DM, Martin M. Validation of the United States' version of the World Health Organization quality of life (WHOQOL) instrument. J Clin Epidemiol. 2000;53(1):1-12. doi:10.1016/S0895-4356(99)00123-7

27. Per BVN. The WHO quality of life (WHOQOL) questionnaire: danish validation study. Nord J Psychiatry. 2001;55(4):229-235. doi: $10.1080 / 080394801681019075$

28. Skevington SM, Carse MS, Williams A. Validation of the WHOQOL-100: pain management improves quality of life for chronic pain patients. Clin J Pain. 2001;17(3):264-275. doi:10.10 97/00002508-200109000-00013
29. Ware JE Jr, Gandek B. Methods for testing data quality, scaling assumptions, and reliability: the IQOLA Project approach. J Clin Epidemiol. 1998;51(11):945-952. doi:10.1016/S0895-4356(98)00 085-7

30. Padrão MB, Sens YA. Quality of life of living kidney donors in Brazil: an evaluation by the short form-36 and the WHOQOL-bref questionnaires. Clin Transplant. 2009;23(5):621-627. doi:10.1111/ j.1399-0012.2009.01048.x

31. Lin M-R, Hwang H-F, Chen C-Y, Chiu W-T. Comparisons of the brief form of the World Health Organization Quality of Life and Short Form-36 for persons with spinal cord injuries. Am J Phys Med Rehabil. 2007;86(2):104-113. doi:10.1097/01.phm.00002477 80.64373.0e

32. Ciconelli RM, Ferraz MB, Santos W, Meinão I, Quaresma MR. Tradução para a língua portuguesa e validação do questionário genérico de avaliação de qualidade de vida SF-36 (Brasil SF-36). Rev Bras Reumatol. 1999;39(3):143-150.

33. Fleck M, Louzada S, Xavier M, et al. Aplicação da versão em português do instrumento abreviado de avaliação da qualidade de vida" WHOQOL-bref". Revista de Saúde Pública. 2000;34:17 8-183. doi:10.1590/S0034-89102000000200012

34. Najafi M, SHEYKH VM, Montazeri A, Abbasi SHA, Sheykh FEM. Quality of Life in Coronary Artery Disease: SF-36 Compared to WHOQOL-BREF. 2008.

35. Unalan D, Soyuer F, Ozturk A, Mistik S. Comparison of SF-36 and WHOQOL-100 in patients with stroke. Neurol India. 2008;56(4):426. doi:10.4103/0028-3886.44573

36. Castro PC, Driusso P, Oishi J. Convergent validity between SF-36 and WHOQOL-BREF in older adults. Rev Saude Publica. 2014;48:63-67. doi:10.1590/S0034-8910.2014048004783
International Journal of General Medicine

\section{Publish your work in this journal}

The International Journal of General Medicine is an international, peer-reviewed open-access journal that focuses on general and internal medicine, pathogenesis, epidemiology, diagnosis, monitoring and treatment protocols. The journal is characterized by the rapid reporting of reviews, original research and clinical studies across all disease areas. The manuscript management system is completely online and includes a very quick and fair peer-review system, which is all easy to use. Visit http://www.dovepress.com/ testimonials.php to read real quotes from published authors. 